\title{
Smooth adiabatic evolutions with leaky power tails
}

\author{
J E Avron and A Elgart 团 \\ Department of Physics, Technion, 32000 Haifa, Israel.
}

Tosio Kato-in memoriam

\begin{abstract}
Adiabatic evolutions with a gap condition have, under a range of circumstances, exponentially small tails that describe the leaking out of the spectral subspace. Adiabatic evolutions without a gap condition do not seem to have this feature in general. This is a known fact for eigenvalue crossing. We show that this is also the case for eigenvalues at the threshold of the continuous spectrum by considering the Friedrichs model.
\end{abstract}

\section{Introduction}

Adiabatic theorems describe the solutions of initial value problems where the Hamiltonian generating the evolution depends slowly on time. In quantum mechanics the description is in terms of spectral information of the instantaneous Hamiltonian. A few basic references on various types of adiabatic theorems are [4, 0, 10, 12, 15, 16].

To formulate the problem more precisely it is convenient to replace the physical time $t$ by the scaled time $s=t / \tau$. One is then concerned with the solution of the initial value problem

$$
i \dot{\psi}_{\tau}(s)=\tau H(s) \psi_{\tau}(s),
$$

with initial data $\psi_{\tau}(0) \in \operatorname{Range} P(0)$. $P(s)$ is a spectral projection for $H(s)$, a selfadjoint Hamiltonian which depends sufficiently smoothly on $s . \psi_{\tau}$ is a vector valued function and the adiabatic limit is the limit of large $\tau$. Suppose, for the sake of concreteness, that $\dot{H}(s)$ is compactly supported on $[0,1]$. As $s$ runs on the interval $[0,1]$, $H(s)$ evolves slowly in physical time for a long interval of time. The total variation of $H(s)$ is finite and not necessarily small.

Adiabatic theorems fall into two baskets: Those that describe the solutions for all times, including times $s \in[0,1]$, and those that characterize the solutions at large times $s>1$ where the Hamiltonian is time independent again. Interestingly they give more precision for long times. We call the first basket, the one that applies to all times, uniform, the second is the long time basket.

$\dagger$ E-mail addresses: avron@physics.technion.ac.il and elgart@physics.technion.ac.il 
Adiabatic theorems can also be put in two other baskets. Those that satisfy a gap conditions and those that do not. By a gap condition we mean a spectral condition on the Hamiltonian $H(s)$ so that a finite gap separates the spectral subspace of the initial data $(=$ Range $P(s))$, from the rest of the spectrum for all times $s$.

A representative result from the uniform basket is [1, 4]:

Theorem 1 Suppose that $P(s)$ is smooth finite rank spectral projection, for a bounded, smooth Hamiltonian $H(s)$. Then, the evolution of the initial state $\psi_{\tau}(0) \in$ RangeP $(0)$, is such that

$$
\operatorname{dist}\left(\psi_{\tau}(s), \text { RangeP }(s)\right) \leq o(1)
$$

for all $s \geq 0$. If, in addition, $P(s)$ satisfies a gap condition then

$$
\operatorname{dist}\left(\psi_{\tau}(s), \operatorname{Range} P(s)\right) \leq O(1 / \tau)
$$

for all $s \geq 0$.

Remark 1 By o(1) we mean a term that vanishes as $\tau \rightarrow \infty$.

Remark 2 Schrödinger operators is a class of application not covered by the theorem because of the assumption that $H(s)$ is bounded. This restriction can be lifted by standard machinery see e.g. [1, 4]. We chose not to enter into this because the essence of the adiabatic evolution is an infrared problem which is largely divorced from the issue of unboundedness of the generator (an ultra-violet problem).

A characteristic result which lies both in the long time basket and in the gap condition basket is [3, 13, 6, 14, 17, 9, 11]:

Theorem 2 Suppose that $H(s)$ is a smooth, bounded, and self-adjoint with $\dot{H}(s)$ supported on $[0,1]$. And suppose that $P(s)$ is separated by a finite gap from the rest of the spectrum. Then, the evolution of the initial state $\psi_{\tau}(0) \in \operatorname{RangeP}(0)$, is such that for any $s>1$,

$$
\operatorname{dist}\left(\psi_{\tau}(s), \operatorname{Range} P(s)\right)=o\left(\frac{1}{\tau^{n}}\right)
$$

for all $n \geq 0$.

Remarks

(i) There is, in general, no uniformity in $n$; the term on the right hand side is of order $\frac{c_{n}}{\tau^{n}}$ where $c_{n}$ are allowed to grow rapidly with $n$.

(ii) In the case where $\dot{H}(s)$ is an analytic family which decays at infinity, the leaking at $s=\infty$ is exponentially small.

(iii) In the case of a gap the distinction between the uniform and the long time basket has an analog in integrals. Suppose that $g(s)$ is real, smooth, strictly monotonic and compactly supported in $[0,1]$. Then

$$
\int_{-\infty}^{s} g(t) e^{i t \tau} d t= \begin{cases}o\left(1 / \tau^{n}\right) & \text { if } s>1, \text { for all } n \\ O(1 / \tau) & \text { if } s \in[0,1]\end{cases}
$$

The gapless basket seems to have no analog in integrals, as we shall see. 
What can one say about adiabatic theorems that lie both in the long time and gapless baskets? In the uniform adiabatic theorem 1, the price one pays for the absence of a gap is, in general, loss of information on the rate at which the adiabatic limit is approached. The question we address here is what is the price one pays in the long time adiabatic theorem, for the absence of a gap.

Adiabatic theorems without a gap condition are normally associated with two distinct settings: Those that describe eigenvalue crossings and those associated with eigenvalue at the threshold of the essential spectrum.

Hagedorn [8] studied adiabatic theorems for crossing eigenvalues. He showed that there is no improvement in the adiabatic theorem in long time limit. In particular, the leaking is always of order $\frac{1}{\sqrt{\tau}}$ for linear crossing.

Here we consider the cases where the absence of a gap is associated with an eigenvalue at the threshold of the continuous spectrum. The results of Hagedorn for crossing eigenvalues do not shed light on this case $\$$.

To investigate this we consider the Friedrichs model [5] with an eigenvalue at the threshold of the continuous spectrum. The model is parameterized by a real parameter $\beta>0$ related to the behavior of the density of states at low energies. Our main result is[

$$
\operatorname{dist}\left(\psi_{\tau}(s), \text { RangeP }(s)\right)= \begin{cases}O\left(\tau^{-\beta}\right) & 1<\beta<2 \text { and } s>1 \\ O\left(\tau^{-1}\right) & 1<\beta<2 \text { and } s \in[0,1]\end{cases}
$$

Unlike the gap basket, the long time behavior in the Friedrichs model indeed has power tails, and unlike the crossing basket, there is an improvement in the rate of decay at long times.

The main application of adiabatic theorems with eigenvalues at threshold is to the theory of atoms in radiation field, where the photon field eliminates spectral gaps. The absence of a gap makes the adiabatic theory of atoms in radiation field qualitatively different from quantum mechanics. Two distinct processes lead to the error in adiabatic evolutions. The first is photon production and the second is atomic excitation. Folk wisdom is that the leaking due to atomic excitations has exponential tails. A simple argument suggests that the leaking due to photon production is of order $1 / \tau$. The argument goes as follows: The power radiated by a dipole is, by classical electrodynamics,

$$
\frac{2}{3} \frac{(\dot{d})^{2}}{c^{3}}
$$

where $d$ is the dipole moment and $c$ the velocity of light. It follows that the number of radiated photons (assuming characteristic frequency of order $O(1 / \tau)$ ), is of order

$$
\left(\frac{\alpha^{3 / 2}}{\tau}\right)^{2}
$$

$\ddagger$ At crossing the spectral projection is discontinuous while for the eigenvalue at threshold that we consider the spectral projection is smooth for all times.

$\S$ All estimates should be considered in asymptotic sense with respect to $\tau \rightarrow \infty$. 
in atomic units!m.

\section{Adiabatic evolutions}

A basic strategy for studying adiabatic evolutions, introduced by Kato [12], is to compare the true evolution, $U_{\tau}(s)$ with a fictitious one, $U_{a}(s)$, which respects the spectral splitting. That is

$$
P(s)=U_{a}(s) P U_{a}^{\dagger}(s) .
$$

We shall denote by a subscript the generator and the evolution of such a fictitious dynamics, i.e.

$$
i \dot{U}_{a}(s)=\tau H_{a}(s) U_{a}(s), U_{a}(0)=1,
$$

while for the true dynamics:

$$
i \dot{U}_{\tau}(s)=\tau H(s) U_{\tau}(s), U_{\tau}(0)=1 .
$$

It was shown by Kato that $H_{a}$ satisfies the commutator equation:

$$
\tau\left[H_{a}(s), P(s)\right]=i[\dot{P}(s), P(s)] .
$$

This commutator equation does not have a unique solution, and different choices of $H_{a}$ can be made. Kato chose:

$$
H_{K}=\frac{i}{\tau}[\dot{P}(s), P(s)] .
$$

This generates a geometric evolution so that, in particular, $U_{K}(s)$ is independent of $\tau$. It is a convenient choice for proving a uniform adiabatic theorem when $P(s)$ is the kernel of $H(s)$. It is inappropriate for generating a systematic adiabatic expansion.

A more effective choice, introduced in [2], is:

$$
H_{A D}=H(s)+\frac{i}{\tau}[\dot{P}(s), P(s)] .
$$

This generator is close to the Hamiltonian and it can be used to prove stronger adiabatic theorems than those handled by Kato, and it can also be used to generate an adiabatic expansion and prove long time adiabatic theorems [13]. However, it is often difficult to use this evolution for concrete computations because there is no explicit formula for $U_{A D}(s ; \tau)$. This is what one needs to do when one wants to show fat leaky tails where one needs a lower bound on the part that leaks. For this reason we shall need yet another choice of an adiabatic evolution that more readily lends itself to explicit computation.

In the special case when the time dependence enters through the unitary family

$$
H(s)=V(s) H V^{\dagger}(s),
$$

the evolution in the rotating frame is both adiabatic and explicit:

$$
U_{r}(s)=V(s) \exp (-i \tau s H) .
$$

$\|$ Recall that in atomic units $\alpha=1 / c$. 
It is generated by

$$
H_{r}(s)=H(s)+\frac{i}{\tau} \dot{V}(s) V^{\dagger}(s),
$$

and like $H_{A D}$ is also close to $H(s)$. This evolution turns out to be inferior to $H_{A D}$ when the game is to prove upper bounds on the leaky tails, but, it is a useful evolution in estimating fat leaky tails where the game is to get lower bounds. The best of all worlds is when $H_{A D}$ and $H_{r}$ coincide. This will turn out to be the case in the Friedrichs model we consider and this is what makes the analysis of this case simple.

\section{The Wave Operator}

To compare the true dynamics with the fictitious dynamics one introduces the wave operator

$$
\Omega_{A D}(s ; \tau)=U_{A D}^{\dagger}(s ; \tau) U_{\tau}(s) .
$$

The leaking out of the spectral subspace is governed by the "off diagonal" part of $\Omega_{A D}$. Namely, from estimate on $P \Omega_{A D}(s ; \tau) P_{\perp}$ and $P_{\perp} \Omega_{A D}(s ; \tau) P$ where $P=P(0)$ and $P_{\perp}=1-P$. This follows from

$$
\begin{aligned}
\left\|P_{\tau}(s)-P(s)\right\| & =\left\|P_{\perp} \Omega_{A D}(s ; \tau) P-P \Omega_{A D}(s ; \tau) P_{\perp}\right\| \\
& =\max \left\{\left\|P_{\perp} \Omega_{A D}(s ; \tau) P\right\|,\left\|P \Omega_{A D}(s ; \tau) P_{\perp}\right\|\right\},
\end{aligned}
$$

where

$$
P_{\tau}(s)=U_{\tau}(s) P U_{\tau}^{\dagger}(s) .
$$

$\Omega_{A D}$ can be calculated via a Volterra-type equation:

$$
\dot{\Omega}_{A D}(s ; \tau)=K_{A D}(s) \Omega_{A D}(s ; \tau),
$$

where

$$
K_{A D}(s)=-U_{A D}^{\dagger}(s ; \tau)[\dot{P}, P](s) U_{A D}(s ; \tau) .
$$

By standard arguments, the series

$$
\Omega_{A D}(s ; \tau)=\sum_{0}^{\infty} \Omega_{i}(s), \quad \Omega_{0}(s)=1, \quad \Omega_{i+1}(s)=\int_{0}^{s} K_{A D}(t) \Omega_{i}(t) d t
$$

is absolutely convergent

It is not a-priori clear that the series in Eq (20) is an expansion in the small parameter of the adiabatic limit, $1 / \tau$. To see that it does note:

\section{Lemma 1}

(i) $\Omega_{2 j}$ is diagonal in the sense that it maps Range $P$ to Range $P$ and Range $P_{\perp}$ to Range $P_{\perp}$, at the same time $\Omega_{2 j+1}$ is off diagonal in the sense that it maps Range $P$ to Range $P_{\perp}$ and Range $P_{\perp}$ to Range $P$. In particular, only the odd terms contribute to leaking.

If Recall that we assume $H(s)$, and $\dot{P}(s)$ bounded. 
Smooth adiabatic evolutions with leaky power tails

(ii) Let us denote $Q(s):=1-\Omega_{A D}(s), f(\tau):=\sup _{s}\|Q(s)\|$. Then for all $s$,

$$
\left\|\Omega_{i+2}(s)\right\|<C f(\tau) \sup _{s}\left\|\Omega_{i}(s)\right\| \text {. }
$$

In particular

$$
\begin{gathered}
\left\|\Omega_{j}(s)\right\|<C f(\tau), \quad j=1,2 ; \\
\left\|\Omega_{j}(s)\right\|<C f^{2}(\tau), \quad j \geq 3 .
\end{gathered}
$$

Remarks

(i) In the case where a gap condition holds, the uniform adiabatic theorem says that $f(\tau)=O(1 / \tau)$. It follows that $\sup _{s}\left\|\Omega_{2 i+1}(s)\right\| \leq \frac{C}{\tau^{i+1}}$. In the absence of a gap condition $f(\tau)$ may, in general, decay more slowly with $\tau$.

(ii) For the lemma to be useful one needs a strong version of the uniform adiabatic theorem which guarantees that $Q(s)$ is small for large $\tau$. This goes beyond the information given by the uniform adiabatic theorem quoted in the previous section. That is, the adiabatic evolution must approximate the true evolution both on Range $P$ and on its complement Range $P_{\perp}$.

(iii) Note that from the estimates above it follows, that when $\Omega_{1}(s)>>f^{2}(\tau)$ for $s>1$ then the leaky tails are determined by $\Omega_{1}(s)$.

Proof The first part of the lemma is standard [2] and follows from the fact that $[\dot{P}, P]$ if off diagonal. The second part follows from the identity:

$$
\begin{aligned}
\Omega_{i+2}(s) & =\int_{0}^{s} Q(t) K_{A D}(t) \Omega_{i}(t) d t-\int_{0}^{s} K_{A D}(t) \Omega_{A D}(t) Q^{\dagger}(t) \Omega_{i+1}(t) d t \\
& -Q(s) \Omega_{i+1}(s) .
\end{aligned}
$$

$K_{A D}(s)$ vanishes outside the interval $[0,1]$, hence,

$$
\begin{aligned}
\left\|\Omega_{i+2}(s)\right\| & \leq f(\tau) \sup _{s}\left(\left\|K_{A D}(s)\right\| \cdot\left\|\Omega_{i}(s)\right\|+\left(\left\|K_{A D}(s)\right\|+1\right)\left\|\Omega_{i+1}(s)\right\|\right) \\
& <2 f(\tau)\left(\sup _{s}\left\|K_{A D}(s)\right\|+1\right) \sup _{s}\left\|\Omega_{i}(s)\right\|
\end{aligned}
$$

\section{Power Tails in Friedrichs Models}

The Friedrichs model $\square$ is defined on the Hilbert space $\mathcal{H}=\mathbb{C} \oplus L^{2}\left(\mathbb{R}_{+}, d \mu(k)\right)$ with inf $($ support $\mu) \geq 0$ and $\mu(0)=0$. A vector $\Psi \in \mathcal{H}$ is normalized by

$$
\Psi=\left(\begin{array}{c}
\omega \\
\psi(k)
\end{array}\right), \quad\|\Psi\|^{2}=|\omega|^{2}+\int_{\mathbf{R}_{+}}|\psi(k)|^{2} d \mu(k), \quad \omega \in \mathbb{C} .
$$

The Friedrichs Hamiltonian $H$ acts on $\mathcal{H}$ like:

$$
H \Psi=\left(\begin{array}{cc}
0 & 0 \\
0 & k
\end{array}\right)\left(\begin{array}{c}
\omega \\
|\psi\rangle
\end{array}\right)=\left(\begin{array}{c}
0 \\
|k \psi\rangle
\end{array}\right) .
$$

+ In some circles this is known as the Fanno model. 
It has a ground state at threshold and the projection on the ground state $P$ is

$$
P=\left(\begin{array}{ll}
1 & 0 \\
0 & 0
\end{array}\right) \text {. }
$$

We consider the case when the time dependence is of the form $H(s)=V(s) H V^{\dagger}(s)$ with unitary $V(s)$ generated by

$$
\dot{V}(s) V^{\dagger}(s)=[\dot{P}(s), P(s)]=i \dot{g}(s)\left(\begin{array}{ll}
0 & \langle\varphi| \\
|\varphi\rangle & 0
\end{array}\right),
$$

where $0 \leq \dot{g}(s) \in C_{0}^{\infty}([0,1])$, and

$$
\int_{0}^{x}|\varphi|^{2} d \mu(k)=O\left(x^{2 \beta}\right), \quad \beta \geq 0
$$

for small $x$. Note that due to the particular form of time dependence (Eq. (28)) one has that $H_{A D}=H_{r}$.

We now borrow a results from [1]:

Proposition 1 For the Friedrichs model and the adiabatic evolution generated by $H_{A D}$, $f(\tau)$ of lemma 1 is such that

$$
f(\tau) \leq \begin{cases}O\left(\frac{1}{\tau}\right), & \beta>1 \\ O\left(\frac{\log \tau}{\tau}\right), & \beta=1 \\ O\left(\tau^{-\beta}\right), & \beta<1\end{cases}
$$

for all $s$.

Now, we come to the main result of this section:

Proposition 2 For the Friedrichs model the evolution of the state that starts as the bound state $\psi_{\tau}(0) \in$ Range $P$, is such that for any $\epsilon>0$

$$
C_{1} \tau^{-\beta+\epsilon}>\operatorname{dist}\left(\psi_{\tau}(s), \operatorname{Range} P(s)\right)>C_{2} \tau^{-\beta},
$$

when $2>\beta>0$ and $s>1$.

Proof With the choice of $H_{A D}$ all the even terms $\Omega_{2 j}$ are diagonal while all the odd terms are off diagonal. By Lemma (1), $\Omega_{j}=O\left(f^{2}(\tau)\right)$ for $j \geq 3$. We need to compute $\Omega_{1}(s)$ and provided it dominates $f^{2}(\tau)$, we are done. Now, for $s>1$,

$$
\begin{aligned}
& P_{\perp} \Omega_{1}(s) P=P_{\perp} \int_{0}^{1} K(t) d s P \\
= & i\left(\begin{array}{ll}
0 & 0 \\
0 & 1
\end{array}\right) \int_{0}^{1} \dot{g}(s) \exp \left(i \tau s\left(\begin{array}{ll}
0 & 0 \\
0 & k
\end{array}\right)\right)\left(\begin{array}{ll}
0 & \langle\varphi| \\
|\varphi\rangle & 0
\end{array}\right)\left(\begin{array}{ll}
1 & 0 \\
0 & 0
\end{array}\right) d s \\
= & i \int_{0}^{1} \dot{g}(s)\left(\begin{array}{ll}
0 & 0 \\
|\exp (i \tau s k) \varphi\rangle & 0
\end{array}\right) d s \\
= & i\left(\begin{array}{ll}
0 & 0 \\
|\hat{g}(\tau k) \varphi\rangle & 0
\end{array}\right)
\end{aligned}
$$


where $\widehat{\dot{g}}$ stands for Fourier transform of $\dot{g}$. Now, since $\dot{g}$ is positive $|\hat{\dot{g}}(k)|$ takes its maximal value at the origin. Since this function is continuous, for some $a, b$ positive $|\widehat{\dot{g}}(k)|>b$ for $k \in[0, a]$. We can estimate now the norm of $P_{\perp} \Omega_{1}(s) P$ from below using Eq. (29):

$$
\begin{aligned}
\left\|P_{\perp} \Omega_{1}(s) P\right\|^{2} & =\| \widehat{\hat{g}}(\tau k) \varphi(k)\rangle \|^{2}=\int_{0}^{\infty}|\widehat{\dot{g}}(\tau k)|^{2}|\varphi(k)|^{2} d \mu(k) \\
& >\int_{0}^{a}|\widehat{\dot{g}}(k)|^{2}\left|\varphi\left(\frac{k}{\tau}\right)\right|^{2} d \mu\left(\frac{k}{\tau}\right) \\
& >b^{2} \int_{0}^{\frac{a}{\tau}}|\varphi(k)|^{2} d \mu(k) \\
& =O\left(\frac{1}{\tau^{2 \beta}}\right), \quad s>1 .
\end{aligned}
$$

Similarly,

$$
\begin{aligned}
\left\|P_{\perp} \Omega_{1}(s) P\right\|^{2} & =\int_{0}^{\infty}|\widehat{\dot{g}}(\tau k)|^{2}|\varphi(k)|^{2} d \mu(k) \\
& =\left\{\int_{0}^{\frac{1}{\tau^{1-\epsilon}}}+\int_{\frac{1}{\tau^{1-\epsilon}}}^{\infty}\right\}|\widehat{\dot{g}}(\tau k)|^{2}|\varphi(k)|^{2} d \mu(k) \\
& <C\left(\frac{1}{\tau^{2 \beta(1-\epsilon)}}+\left|\widehat{\dot{g}}\left(\tau^{\epsilon}\right)\right|^{2}\right), \quad s>1 .
\end{aligned}
$$

Since $|\hat{\dot{g}}(k)|$ is decaying faster then any power at large $k$, the proof is complete.

Note that there is an improvement in the long time limit over the uniform result for $2>\beta \geq 1$.

Acknowledgment We thank M.V. Berry for several useful conversations. This research was supported in part by the Israel Science Foundation, the Fund for Promotion of Research at the Technion and the DFG.

\section{Appendix A. Slaved Leaking}

In this appendix we want to address an apparent puzzle associated with the (standard) long time adiabatic theorem.

It is, in fact, surprising that a gap condition is all one needs for the fast decay in the long time adiabatic theorem: Suppose that $H(s)$ had one eigenvalue separated by a gap from the rest of the spectrum, which is purely absolutely continuous. For time $s \in[0,1]$ the leaking to the (instantaneous) absolutely continuous spectrum is $O(1 / \tau)$. One would expect that once a piece leaks to the absolutely continuous part of the spectrum it would propagate to infinity, as states in the absolutely continuous spectrum invariably do. If this was the case, then it would be difficult to understand how the wave reconstructs itself so that, for times $s>1$, the part in the absolutely continuous spectrum is smaller than any power in $1 / \tau$. As we shall see from the proof of the adiabatic theorem to all

orders below, the parts that leaks to the absolutely continuous spectrum and is a finite power of $\tau$ is slaved to the instantaneous eigenvalue, and disappears for time $s>1$. It 
Smooth adiabatic evolutions with leaky power tails

does not propagate. Only the terms smaller than any power in $1 / \tau$ are free to propagate to infinity.

The following lemma is taken, verbatim, from 18

Lemma 2 Let $R(s, z)$ be the resolvent of $H(s)$ and define the tilde operation by

$$
\tilde{X}(s)=-\frac{1}{2 \pi i} \int_{\Gamma} R(s, z) X(s) R(s, z) d z
$$

where $\Gamma$ is a contour enclosing the part of the spectrum associated with $P(s)$. Let, $X(s)$ and $Y(s)$ be bounded, then

$$
\begin{aligned}
& P_{\perp} \int_{0}^{s} U_{A D}^{\dagger}(t) X(t) U_{A D} P Y(t) d t=\frac{i}{\tau} P_{\perp}\left(\left.U_{A D}^{\dagger}(t) \tilde{X}(t) U_{A D} P Y(t)\right|_{0} ^{s}\right. \\
& \left.-\int_{0}^{s} U_{A D}^{\dagger}(t) \dot{\tilde{X}}(t) U_{A D} P Y(t) d t-\int_{0}^{s} U_{A D}^{\dagger}(t) \tilde{X}(t) U_{A D} P \dot{Y}(t) d t\right)
\end{aligned}
$$

If we now apply the lemma with $X(s)=[\dot{P}, P](s)$ to $\Omega_{j+1}$ we get

$$
\begin{aligned}
P_{\perp} \Omega_{j+1}(s) & =P_{\perp} \int_{0}^{s} U_{A D}^{\dagger}(t) X(t) U_{A D} P \Omega_{j}(t) d t \\
& =-\frac{i}{\tau} P_{\perp}\left(U_{A D}^{\dagger}(s) \tilde{X}(s) U_{A D}(s) P \Omega_{j}(s)\right. \\
& -\int_{0}^{s} U_{A D}^{\dagger}(t) \dot{\tilde{X}}(t) U_{A D} P \Omega_{j}(t) d t \\
& \left.-\int_{0}^{s} U_{A D}^{\dagger}(t) \tilde{X}(t) U_{A D} P K(t) \Omega_{j-1}(t) d t\right)
\end{aligned}
$$

Each of the integrals in the above expression is once again of the form so that the lemma can be applied again and again. We see that the power terms that occur for $0<s<1$

are boundary terms, proportional to $\dot{P}(s)$, and instantaneously reflect what happens to the bound state. That is, the power tails in Range $P_{\perp}(s)$ are slaved to the state in Range $P(s)$.

\section{Appendix B. Asymptotics of Fourier Transforms in $C_{0}^{\infty}$}

When $g \in C_{0}^{\infty}$ its Fourier transform decays faster than any power, but not quite exponentially. The canonical example of such a function is

$$
g(s)=\exp \left(\frac{-1}{(1-s)(1+s)}\right), \quad s \in[-1,1]
$$

and zero otherwise. It is of some interest to have an explicit asymptotic expansion of its Fourier transform.

Lemma $3 \widehat{g}(p)$ is an entire even function of $p$, and on the real p-axis it has the asymptotic behavior:

$$
\widehat{g}(p)=2 \frac{\sqrt{\pi}}{(2 e)^{1 / 4}} \cdot \frac{\exp (-\sqrt{p})}{p^{3 / 4}} \cdot\left(\cos \left(p-\sqrt{p}-\frac{3}{8} \pi\right)+O(1 / \sqrt{p})\right) .
$$


Proof That the function is even and entire follows directly from the definition

$$
\widehat{g}(p)=\int_{-1}^{1} \exp (i p s) \cdot \exp \left(\frac{-1}{1-s^{2}}\right) d s=\int_{-1}^{1} \cos (p s) \cdot \exp \left(\frac{-1}{1-s^{2}}\right) d s .
$$

The asymptotics follows from Laplace saddle point method. Let

$$
h(s, p)=-\frac{1}{1-s^{2}}+i s p,
$$

denote the logarithm of the integrand. The integral is estimated by Gaussian integrals near the appropriate extrema of $h$. The extremum at $s_{0}$ contributes

$$
\sqrt{\frac{2 \pi}{-h^{\prime \prime}\left(s_{0}, p\right)}} \cdot \exp h\left(s_{0}, p\right)
$$

and prime denotes a partial derivative with respect to $s$.

The extrema are the solutions of the quartic equation

$$
h^{\prime}(s, p)=-\frac{2 s}{\left(1-s^{2}\right)^{2}}+i p=0 .
$$

When $p$ is large two of the solutions are close to 1 and the other two are close to -1 . To leading order in $p$ the two extrema near 1 are

$$
s_{0}(p)=(1 \pm \delta(p)), \quad \delta(p)=\frac{1}{\sqrt{2 i p}}+O\left(\frac{1}{p^{3 / 2}}\right)
$$

where

$$
h(1 \pm \delta, p)=i p \pm(1+i) \sqrt{p}-\frac{1}{4}+O(\delta) .
$$

It is now clear that the right way to deform the contour is so that it goes through $1-\delta$ and the saddle point at $1+\delta$ should be avoided. Now, to leading order in $p$ the second derivative there is

$$
\begin{aligned}
h^{\prime \prime}\left(s_{0}, p\right) & =-\frac{8 s^{2}}{\left(1-s^{2}\right)^{3}}+O(p) \\
& =-(2 i p)^{3 / 2}(1+O(\delta)) \\
& =-(2 p)^{3 / 2} \exp \left(\frac{3 \pi i}{4}\right) \cdot(1+O(\delta))
\end{aligned}
$$

Substituting in Eq. (B.5) one finds the contribution from the saddle points near 1 to be

$$
\frac{\sqrt{\pi}}{(2 e)^{1 / 4}} \cdot \frac{\exp (-\sqrt{p})}{p^{3 / 4}} \cdot \exp (i(p-\sqrt{p}-3 \pi / 8))(1+O(1 / \sqrt{p})) .
$$

Using the fact that $\hat{g}(p)$ is real valued for real $p$, the saddle near -1 must give the complex conjugate of this, and the result follows.

\section{References}

[1] Avron, J. E., Elgart, A.: Adiabatic Theorem without a Gap Condition. Comm. Math. Phys. 203 , 445-463,(1999) 
[2] Avron, J. E., Seiler, R., Yaffe, L. G.: Adiabatic theorems and applications to the quantum Hall effect. Comm. in Math. Phys. 110, 33-49 (1987), (Erratum: Comm. in Math. Phys. 153, 649-650 (1993))

[3] Berry, M.V.: Histories of adiabatic transition. Proc. Roy. Soc. Lond. A 429, 61-72 (1990)

[4] F. Bornemann, Homogenization in time of singularly perturbed mechanical systems, Lecture Notes in Math. 1687, Springer (1998).

[5] Friedrichs, K. O.: On the perturbation of continuous spectra. Comm. Pure Appl. Math. 1, 361-406 (1948)

[6] Garrido, L. M.: Generalized adiabatic invariance. J. Math. Phys. 5, 355-362 1964

[7] Galindo, A., Pascual, P.: Quantum mechanics. Berlin-Heidelberg-New-York: Springer-Verlag, 1991

[8] Hagedorn, G.: Adiabatic Expansions near Eigenvalue Crossings. Ann. Phys. 196, 278-295 1989

[9] Jakšić, V., Segert, J.: On the Landau Zener formula for two-level systems. J. Math. Phys. 34, 2807-2820 1993

[10] Joye, A., Monti, F., Guerin, S., Jauslin, H. R.: Adiabatic Evolution for Systems with Infinitely many Eigenvalue Crossings, to appear in J. Math. Phys., preprint math-ph/9812024

[11] Joye, A., Pfister, C.E.: Exponential Estimates in Adiabatic Quantum Evolution. Proceeding of the XII ICMP, Brisbane Australia (1997); Quantum Adiabatic Evolution. In On Three Levels. Fannes, M., Maes, C., Verbure, A., editors, London: Plenum, 1994

[12] Kato, T.: On the adiabatic theorem of quantum mechanics. Phys. Soc. Jap. 5, 435-439 1950

[13] Klein, M., Seiler, R.: Power law corrections to the Kubo formula vanish in quantum Hall systems. Comm. in Math. Phys. 128, 1411990

[14] Martinez, A.: Precise exponential estimates in adiabatic theory, J. Math. Phys. 35, 3889-3915 1994

[15] Martinez, A., Nakamura, S.: Adiabatic limit and scattering. C.R. Acd. Sci. Paris. 318, 1153-1158 1994

[16] Nenciu, G.: On the adiabatic theorem of quantum mechanics. J. Phys. A 13, L15-L18 1980

[17] Nenciu, G.: Linear Adiabatic Theory: Exponential Estimates. Comm. in Math. Phys. 152, 479-496 1993

[18] Richter, T., Seiler, R.: Geometric Properties of Transport in Quantum Hall Systems, preprint mp-arc 99-226 